\title{
Determining the structure of a biological medium using acoustooptic probes*
}

\author{
H. T. Banks and T. Lin \\ Center for Research in Scientific Computation \\ N.C. State University \\ Raleigh, NC 27695-8205
}

November 12, 1998

\section{Introduction}

Non-invasive interrogating techniques are most valuable in determining substructure in biological tissues due to the fact that they usually result in much less discomfort in subjects. Controlled microwaves (electromagnetic waves in the frequency range of 3 to 300 gigahertz) can pass through many media without causing damage. On the other hand, chemical and physical changes in biological tissue can result in changes in its electromagnetic characteristics such as electric and magnetic polarization mechanisms and conductivity. Consequently, microwaves sent into two different tissues will show different propagation features, and analysis of these features often can yield useful information on tissue dysfunction. Applications of the use of microwaves in non-invasive interrogation procedures can be found in a recently published review article [1]. Use of ultrasonic waves is another popular technique used in non-invasive interrogation of media in both industrial and medical applications. It has been well known since 1922 [5] that electromagnetic and sound waves can interact in a medium and influence each other's propagation. This interaction has been the subject of substantial investigation in acoustooptics [7, 8, 10], and numerous acoustooptic devices have been developed in many applications in industry such as neural nets, optical excision, and fiber optics to name just a few.

In this report, we investigate the possibility of using the interaction between electromagnetic and ultrasonic waves to interrogate the structure of a biological medium. The medium

${ }^{*}$ Research was supported in part by the Air Force Office of Scientific Research under grants AFOSR F49620-95-1-0236 and AFOSR F49620-98-1-0180. T. Lin was also partially supported by the National Science Foundation under grant DMS-9704621. The permanent address of T. Lin is: Department of Mathematics, Virginia Polytechnic Institute and State University, Blacksburg, VA 24060 
considered here is a fluid; this is motivated by the fact that the major component of human tissue is water. We focus on a class of models for electric polarization in the context of Maxwell's equations. It is well known (e.g., see [2,3] and the references therein), that polarization mechanisms will affect substantially the propagation of an electromagnetic wave passing through a medium. Our aim here is to demonstrate how modification of the polarization feature in a tissue by an ultrasonic wave, which produces a virtual interface, can be used. An electromagnetic probe sent into this tissue will partially reflect from this artificial interface, and return information (e.g., geometry) about the part of the tissue between its surface and this artificial interface.

For the propagation of the microwaves, we assume, as in [2, 3], that the Maxwell's equations hold; specifically,

$$
\begin{aligned}
& \nabla \times \mathbf{E}=-\frac{\partial \mathbf{B}}{\partial t}, \\
& \nabla \times \mathbf{H}=\frac{\partial \mathbf{D}}{\partial t}+\mathbf{J} \\
& \nabla \cdot \mathbf{D}=\rho_{e m}, \\
& \nabla \cdot \mathbf{B}=0 .
\end{aligned}
$$

along with the basic constitutive laws for a conductive (Ohm's law) dielectric:

$$
\begin{aligned}
& \mathbf{J}=\sigma \mathbf{E}, \\
& \mathbf{D}=\epsilon_{0} \mathbf{E}+\mathbf{P}, \\
& \mathbf{B}=\mu_{0} \mathbf{H}+\mu_{0} \mathbf{M} .
\end{aligned}
$$

Here $\mathbf{E}$ is the electric field intensity, $\mathbf{D}$ is the (displacement) electric flux density, $\mathbf{H}$ is the magnetic field intensity, $\mathbf{B}$ is the magnetic flux intensity, $\mathbf{J}$ is current density, $\rho_{e m}$ is the free (unpaired) charge density, $\mathbf{P}$ is the electric polarization, $\mathbf{M}$ is the magnetic polarization. In our initial efforts, we concentrate on the propagation of an electromagnetic wave which is uniform in the $x-y$ plane moving in the $z$ direction. The motivation (use of polarized impulsive probes) is explained more fully in $[2,3]$. This assumption allows us to consider the electromagnetic fields in the following form: $\mathbf{E}=E(t, z) \mathbf{i}, \mathbf{P}=P(t, z) \mathbf{i}, \mathbf{H}=H(t, z) \mathbf{j}$. We consider electromagnetic wave propagation in the normalized interval $z \in[0,1]$, assume that the fluid slab occupies the space for $z \in\left[z_{1}, 1\right]$, and an acoustic wave is given in the part of the fluid for $z \in\left[z_{2}, 1\right]$ with $0<z_{1}<z_{2}<1$. Figure 1 is a sketch of the geometry considered here.

Since we are mainly interested in biological media which are non-magnetic, we further assume that the magnetic polarization is zero, i.e., $\mathbf{M}=\mathbf{0}$. Then Maxwell's equations together with the basic constitutive laws yield the following equation in the domain $0<z<$ 1:

$$
\frac{\partial^{2} E}{\partial t^{2}}+\frac{\sigma}{\epsilon_{0}} \frac{\partial E}{\partial t}+\frac{1}{\epsilon_{0}} \frac{\partial^{2} P}{\partial t^{2}}-\frac{1}{\epsilon_{0} \mu_{0}} \frac{\partial^{2} E}{\partial z^{2}}=-\frac{1}{\epsilon_{0}} \frac{\partial J_{s}}{\partial t} .
$$




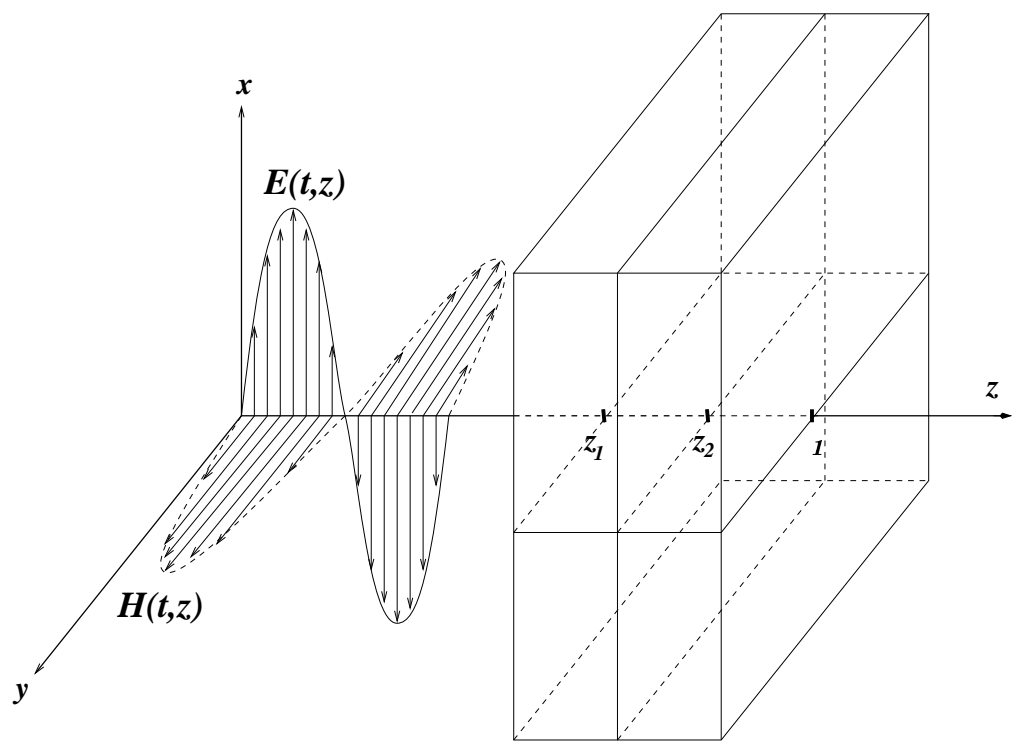

Figure 1: Geometry of physical problem.

Applying an electronic field to a dielectric material will cause electric polarization which is, of course, material dependent. In our investigation here, the part of material for $z \in\left[0, z_{1}\right]$, which is air, is assumed to have zero electric polarization and zero conductivity; the part of material for $z \in\left[z_{1}, z_{2}\right]$ is assumed to obey the Debye law (Chapter 2, [6]), in which the electric polarization responds to the electric field in a decaying first order manner:

$$
\tau \frac{\partial P}{\partial t}+P=\epsilon_{0}\left(\epsilon_{s}-\epsilon_{\infty}\right) E
$$

Here $\epsilon_{s}$ and $\epsilon_{\infty}$ are the static relative permittivity and high frequency relative permittivity, respectively, and $\tau$ is the relaxation time. More details of this model can be found in [6], and other models for the polarization can be found in $[2,3]$ and references there.

The introduction of an acoustic wave will change the density of the fluid (indeed, acoustic waves are simply pressure waves which involve density variations). This in turn will affect electromagnetic properties, such as the refraction index, of the fluid. This is known as the acoustooptic effect. Consequently, any electronic wave transmitted into this part of fluid will be modulated by the acoustic wave. At the same time, the material electrostriction caused by the electronic waves will also affect the propagation of the pressure wave in the fluid [9]. This produces a fully coupled nonlinear model with equations for both the electromagnetic and acoustic pressure waves (see [4] and page 825 of [9]). In our initial efforts, we focus on the effects of the acoustic wave as a reflector of electromagnetic waves. We ignore the effect of electromagnetic forces in the acoustic equation under the tacit assumption that the effect is weak. To demonstrate the effect of the acoustic wave on the electronic wave, we begin with a common assumption [8] that the electric susceptibility is an affine function of the 
acoustic pressure $p(t, z)$ :

$$
\chi=\chi_{0}+\chi_{1} p(t, z)
$$

Then we have

$$
P=\epsilon_{0} \chi E=\epsilon_{0}\left(\chi_{0}+\chi_{1} p(t, z)\right) E,
$$

and hence

$$
\frac{\partial^{2} P}{\partial t^{2}}=\epsilon_{0} \chi_{0} \frac{\partial^{2} E}{\partial t^{2}}+\epsilon_{0} \chi_{1}\left(\frac{\partial^{2} p}{\partial t^{2}} E+2 \frac{\partial p}{\partial t} \frac{\partial E}{\partial t}+p \frac{\partial^{2} E}{\partial t^{2}}\right) .
$$

More generally, we may assume that the fluid in the acoustically effected part of the domain obeys a generalized pressure dependent polarization rule (Chapter 9, [6]):

$$
\frac{1}{\epsilon_{0}} \frac{\partial^{2} P}{\partial t^{2}}=f_{0}(p) E+f_{1}(p) \frac{\partial E}{\partial t}+f_{2}(p) \frac{\partial^{2} E}{\partial t^{2}} .
$$

To simplify issues in this preliminary investigation, we take

$$
f_{0}(p)=0, \quad f_{1}(p)=0, \quad f_{2}(p)=\kappa p(t, z),
$$

which we note is not a special case of (1.10). Thus our polarization assumption in the acoustic fluid is given by

$$
\frac{1}{\epsilon_{0}} \frac{\partial^{2} P}{\partial t^{2}}=\kappa p(t, z) \frac{\partial^{2} E}{\partial t^{2}} .
$$

Using (1.11) in (1.8), we have the following partial differential equation for the electric field in the region disturbed by acoustic waves:

$$
\frac{\partial^{2} E}{\partial t^{2}}+\frac{\sigma}{\epsilon_{0}} \frac{\partial E}{\partial t}+\kappa p(t, z) \frac{\partial^{2} E}{\partial t^{2}}=\frac{1}{\epsilon_{0} \mu_{0}} \frac{\partial^{2} E}{\partial z^{2}}-\frac{1}{\epsilon_{0}} \frac{\partial J_{s}}{\partial t}, \quad z_{2}<z<1 .
$$

This equation is very similar to the one given in [9] derived from thermodynamical considerations.

To complete the demonstration model, we assume that the material outside the space $z \in[0,1]$ can absorb the electronic wave completely; hence we can use the following boundary conditions for the electric field:

$$
\begin{aligned}
& \frac{\partial E}{\partial t}(t, 0)-c \frac{\partial E}{\partial z}(t, 0)=0 \\
& \frac{\partial E}{\partial t}(t, 1)+\frac{c}{\sqrt{1+\kappa p(t, z)}} \frac{\partial E}{\partial z}(t, 1)=0,
\end{aligned}
$$

with

$$
c^{2}=\frac{1}{\epsilon_{0} \mu_{0}} .
$$


We also assume the initial conditions:

$$
E(0, z)=0, \quad \frac{\partial E}{\partial t}(0, z)=0 .
$$

For the purpose of deriving an efficient numerical scheme, we reduce the second order derivative of $P$ in (1.8) by the Debye's polarization law (1.9). This results in a first order derivative of $P$ along with a similar additional term for $E$ in the basic Maxwell's equation. Thus, we use the following initial-boundary value problem to model the dynamics of the electromagnetic fields:

$$
\begin{aligned}
& a(t, z) \frac{\partial^{2} E}{\partial t^{2}}+b(z) \frac{\partial E}{\partial t}+e(z) \frac{\partial P}{\partial t}=d(z) \frac{\partial^{2} E}{\partial z^{2}}+F(t, z), \quad z \in(0,1), \\
& \frac{\partial P}{\partial t}=\eta_{2} P+\gamma_{2} E, \quad z \in\left[z_{1}, z_{2}\right], \\
& \frac{\partial E}{\partial t}(t, 0)-\sqrt{d / a} \frac{\partial E_{1}}{\partial z}(t, 0)=0, \quad \frac{\partial E}{\partial t}(t, 1)+\sqrt{d / a} \frac{\partial E}{\partial z}(t, 1)=0, \\
& E(0, z)=0, \quad \frac{\partial E(0, z)}{\partial t}=0 \\
& P(0, z)=0 .
\end{aligned}
$$

where

$$
\begin{aligned}
& a(t, z)=1+\kappa p(t, z) \chi_{\left[z_{2}, 1\right]}, \\
& b(z)=\frac{\sigma}{\epsilon_{0}} \chi_{\left[z_{1}, 1\right]}+\frac{\gamma_{2}}{\epsilon_{0}} \chi_{\left[z_{1}, z_{2}\right]}, \\
& e(z)=\frac{\eta_{2}}{\epsilon_{0}} \chi_{\left[z_{1}, z_{2}\right]}, \\
& d(z)=c^{2}=\frac{1}{\epsilon_{0} \mu_{0}}, \\
& \eta_{2}=-\frac{1}{\tau}, \quad \gamma_{2}=\frac{\epsilon_{0}\left(\epsilon_{s}-\epsilon_{\infty}\right)}{\tau}, \quad F(t, z)=-\frac{1}{\epsilon_{0}} \frac{\partial J_{s}}{\partial t},
\end{aligned}
$$

and $\chi_{\left[x_{1}, x_{2}\right]}$ is the usual characteristic function for the interval $\left[x_{1}, x_{2}\right]$.

\section{A finite element scheme}

To approximate the solutions to the unknowns $E(t, z)$ and $P(t, z)$ in the model presented in the previous section, we first introduce a partition in the space variable:

$$
0=x_{0}<x_{1}<\cdots<x_{N_{z}}=1 \text {, }
$$

such that

$$
z_{1}=x_{i_{1}}, z_{2}=x_{i_{2}},
$$


for some integers $1<i_{1}<i_{2}<N_{z}$, and

$$
h=\max _{i=1,2, \cdots, N_{z}}\left(x_{i}-x_{i-1}\right) .
$$

Then we let $S^{h} \subset H^{1}(0,1)$ be the standard linear finite element space defined on this partition with a set of basis functions $\left\{\phi_{i}\right\}_{i=0}^{N_{z}}$. We also use a partition in the time variable:

$$
0=t^{0}<t^{1}<\cdots<t^{N_{t}}=T
$$

with $\Delta t=t^{i}-t^{i-1}, \quad i=1, \cdots, N_{t}$.

As usual, we start with the weak form (see $[2,3]$ ) of the model partial differential equation $(1.12)$ :

$$
\begin{gathered}
\left\langle v, a \frac{\partial^{2} E}{\partial t^{2}}\right\rangle+\left\langle v, b \frac{\partial E}{\partial t}\right\rangle+\left\langle v, e \eta_{2} P\right\rangle+\left\langle v, e \gamma_{2} E\right\rangle \\
=-\left.v \sqrt{a d} \frac{\partial E}{\partial t}\right|^{z=1}-\left.v \sqrt{a d} \frac{\partial E}{\partial t}\right|^{z=0}-\left\langle v^{\prime}, d \frac{\partial E}{\partial z}\right\rangle+\langle v, F\rangle,
\end{gathered}
$$

for any $v \in H^{1}(0,1)$, where $\langle$,$\rangle denotes the usual L^{2}(0,1)$ inner product. From this, we can formulate the following finite element discretization of (1.12):

$$
\begin{gathered}
\left\langle\phi_{i}, a \partial_{t t} E^{n}\right\rangle+\left\langle\phi_{i}, b \partial_{t} E^{n}\right\rangle+\left\langle\phi_{i}, e \eta_{2} P^{n}\right\rangle+\left\langle\phi_{i}, e \gamma_{2} E^{n, 1 / 4}\right\rangle=-\left.\sqrt{a d} \partial_{t} E^{n} \phi_{i}\right|^{z=1} \\
-\left.\sqrt{a d} \partial_{t} E^{n} \phi_{i}\right|^{z=0}-\left\langle\phi_{i}^{\prime}, d\left(E^{n, 1 / 4}\right)^{\prime}\right\rangle+\left\langle\phi_{i}, F\left(t^{n}, z\right)\right\rangle
\end{gathered}
$$

where

$$
\begin{aligned}
& E^{n}(z)=\sum_{j=1}^{N} e_{j}^{n} \phi_{j}(z) \approx E\left(t^{n}, z\right), \\
& P^{n}(z)=\sum_{j=1}^{N} p_{j}^{n} \phi_{j}(z) \approx P\left(t^{n}, z\right),
\end{aligned}
$$

and the following finite difference notation is used for the average

$$
U^{n, 1 / 4} \equiv \frac{U^{n+1}+2 U^{n}+U^{n-1}}{4}
$$

along with the difference quotients

$$
\partial_{t t} U^{n} \equiv \frac{U^{n+1}-2 U^{n}+U^{n-1}}{\Delta t^{2}}, \quad \partial_{t} U^{n} \equiv \frac{U^{n+1}-U^{n-1}}{2 \Delta t}
$$

As a scheme to compute $E^{n}$, assuming that $P^{n}$ is given exactly, we expect the above scheme to have the following accuracy:

$$
\left\|E^{n}-E\left(t^{n}, \cdot\right)\right\|_{L^{2}} \leq C\left(h^{2}+\Delta t^{2}\right)
$$


provided that the solution is smooth enough. To update $P^{n}$ at each time step, it is preferable to choose a scheme that has good stability and whose accuracy matches that used to compute $E^{n}$. Hence we use the following A-stable second order Adams-Moulton scheme to discretize the equation (1.13):

$$
P^{n}=\frac{1}{1-\frac{\eta_{2} \Delta t}{2}}\left(P^{n-1}+\frac{\Delta t}{2}\left(\gamma_{2}\left(E^{n}+E^{n-1}\right)+\eta_{2} P^{n-1}\right)\right) .
$$

According to the given initial conditions, we should set

$$
E^{0}=0, \quad P^{0}=0,
$$

for the finite element approximations at the 0-th time level. By the Taylor expansion and applying (1.12), (1.13), (1.15), and (1.16), we have

$$
\begin{aligned}
E\left(t^{1}, z\right) & =E(0, z)+\Delta t \frac{\partial E(0, z)}{\partial t}+\frac{\Delta t^{2}}{2} \frac{\partial^{2} E(0, z)}{\partial t^{2}}+O\left(\Delta t^{3}\right) \\
& \approx \frac{\Delta t^{2}}{2 a(0, z)} F(0, z)
\end{aligned}
$$

Therefore we can let

$$
E^{1}=\frac{\Delta t^{2}}{2 a(0, z)} F(0, z)
$$

Putting all of these discretizations together, we have the following algorithm to generate approximations to both $E(t, z)$ and $P(t, z)$ :

Step 1 . Compute $E^{0}$ and $P^{0}$ by (2.19).

Step 2 . Compute $E^{1}$ by $(2.20)$.

Step 3 . Then for each $n=1,2, \cdots, N_{t}-1$, we use $(2.18)$ to compute $P^{n} \approx P\left(t^{n}, z\right)$ and use (2.17) to compute $E^{n+1} \approx E\left(t^{n+1}, z\right)$.

\section{Some numerical simulations}

To test the model, we assume that a time "windowed" electromagnetic point source input (e.g., see $[2,3]$ ) is given at the left boundary point $z=0$ such that

$$
J_{s}(t, z)=-\delta(z) \chi_{\left[0, t_{f}\right]}(t) \sin \left(\omega_{s} t\right)
$$

The frequency in the source is assumed to be in the microwave range, i.e.,

$$
\omega_{s} \in\left[3 \times 10^{9} \mathrm{~Hz}, 3 \times 10^{11} \mathrm{~Hz}\right] .
$$


The pressure is given by

$$
\kappa p(t, z)=\kappa g(z) \sin \left(\omega_{p} t\right)
$$

such that its frequency is in the ultrasonic range, i.e.,

$$
\omega_{p} \in[0.1 M H z, 25 M H z],
$$

and in all the computations presented here, $g(z)=\chi_{\left[z_{2}, 1\right]}(z)$.

\begin{tabular}{|c|c|c|c|}
\hline$t_{f}$ & $4.0 \times 10^{-10}$ & $\tau$ & $3.162277660168379 \times 10^{-8}$ \\
\hline$\epsilon_{\infty}$ & 5.5 & $\epsilon_{s}$ & 78.2 \\
\hline$\sigma$ & $1.0 \times 10^{-5}$ & $\kappa$ & 10.0 \\
\hline$\omega_{p}$ & $\pi \times 10^{7}$ & $\omega_{s}$ & $\pi \times 10^{10}$ \\
\hline$\Delta t$ & $t_{f} / 1600$ & $h$ & $1 / 900$ \\
\hline
\end{tabular}

Table 1: Parameters used in Example 1.

Example 1: We chose all the parameters in this example similar to those for water except for the relaxation time $\tau$, with the value for $\tau$ used here somewhat larger than those given in the literature. We observed that smaller $\tau$ leads to a weaker transmitted electronic wave into the fluid which hinders observation of the interaction between the electronic and acoustic waves. Some of the parameters are listed in Table 1. Representative plots are in Figure 2-5.

Example 2: The data used in this example are listed in Table 2. All the values are the same as those in the previous example except for the frequency in the acoustic wave. In this case, a lower frequency is used in the acoustic wave and we notice that the reflected wave from the acoustic beam is weaker than that in the previous example, see Figure 6.

\begin{tabular}{|c|c|c|c|}
\hline$t_{f}$ & $4.0 \times 10^{-10}$ & $\tau$ & $3.162277660168379 \times 10^{-8}$ \\
\hline$\epsilon_{\infty}$ & 5.5 & $\epsilon_{s}$ & 78.2 \\
\hline$\sigma$ & $1.0 \times 10^{-5}$ & $\kappa$ & 10.0 \\
\hline$\omega_{p}$ & $\pi \times 10^{6}$ & $\omega_{s}$ & $\pi \times 10^{10}$ \\
\hline$\Delta t$ & $t_{f} / 1600$ & $h$ & $1 / 900$ \\
\hline
\end{tabular}

Table 2: Parameters used for Example 2. 


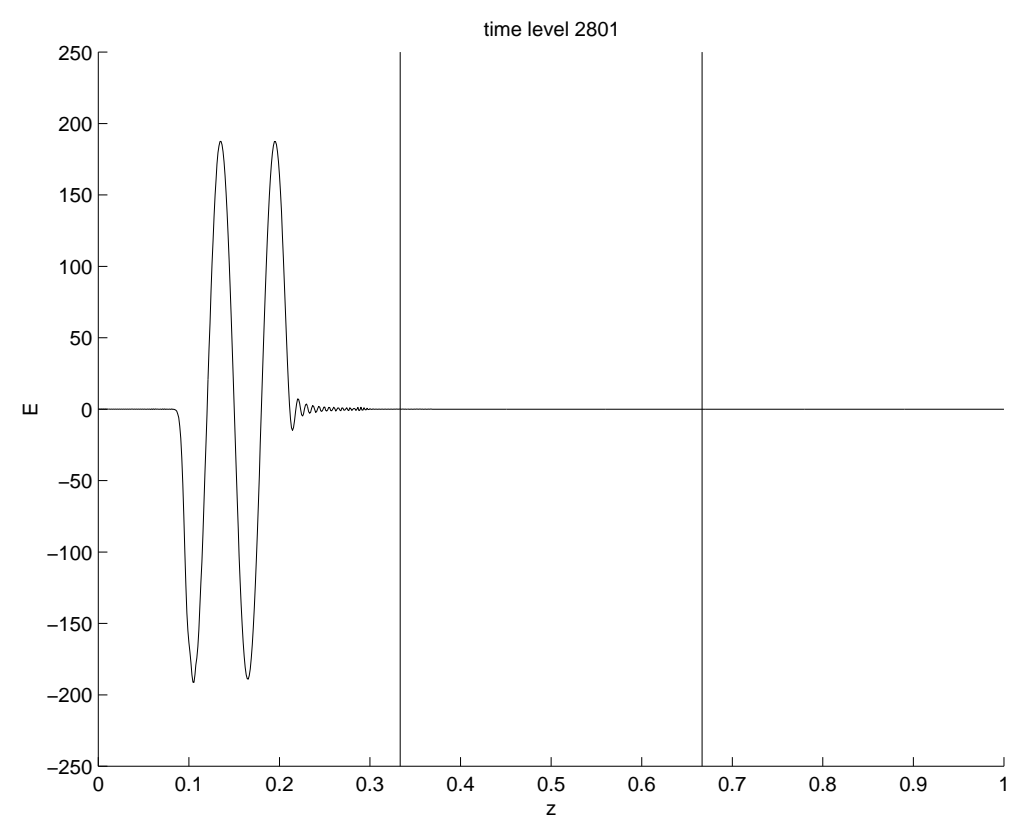

Figure 2: Example 1. The electronic wave before it reaches fluid at $z=z_{1}$.

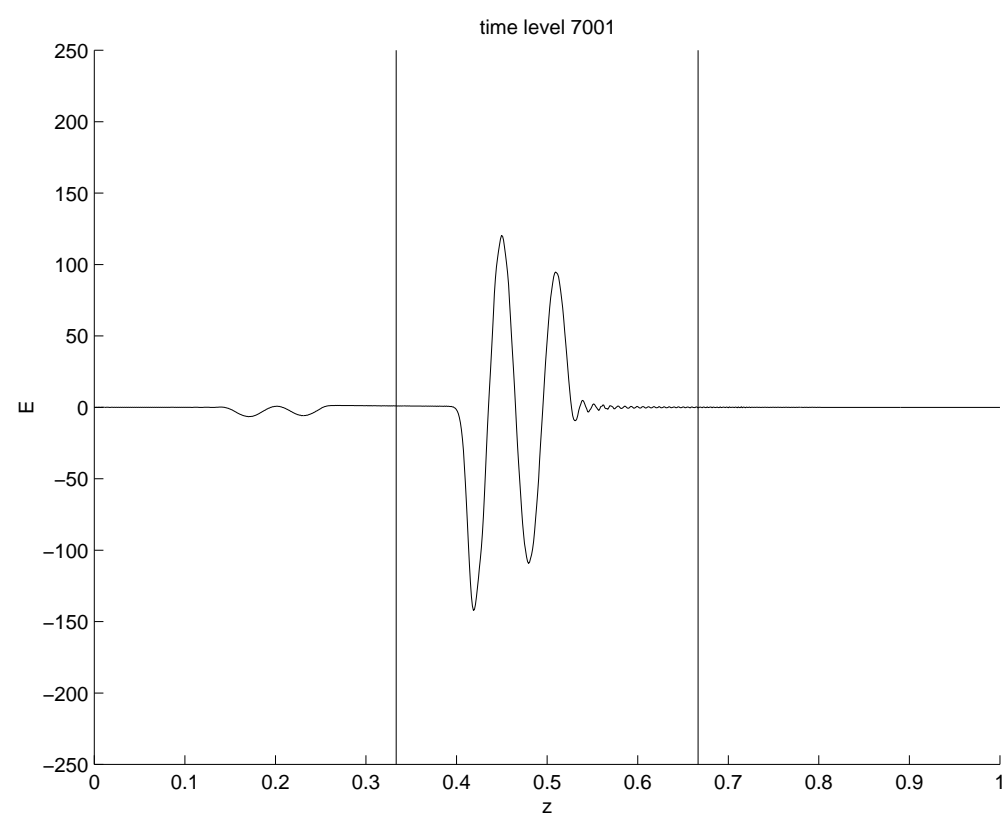

Figure 3: Example 1. The electronic wave in the fluid $\left[z_{1}, z_{2}\right]$ before it interacts with the acoustic wave at $z=z_{2}$. A reflected wave is generated at the interface of air and fluid. 


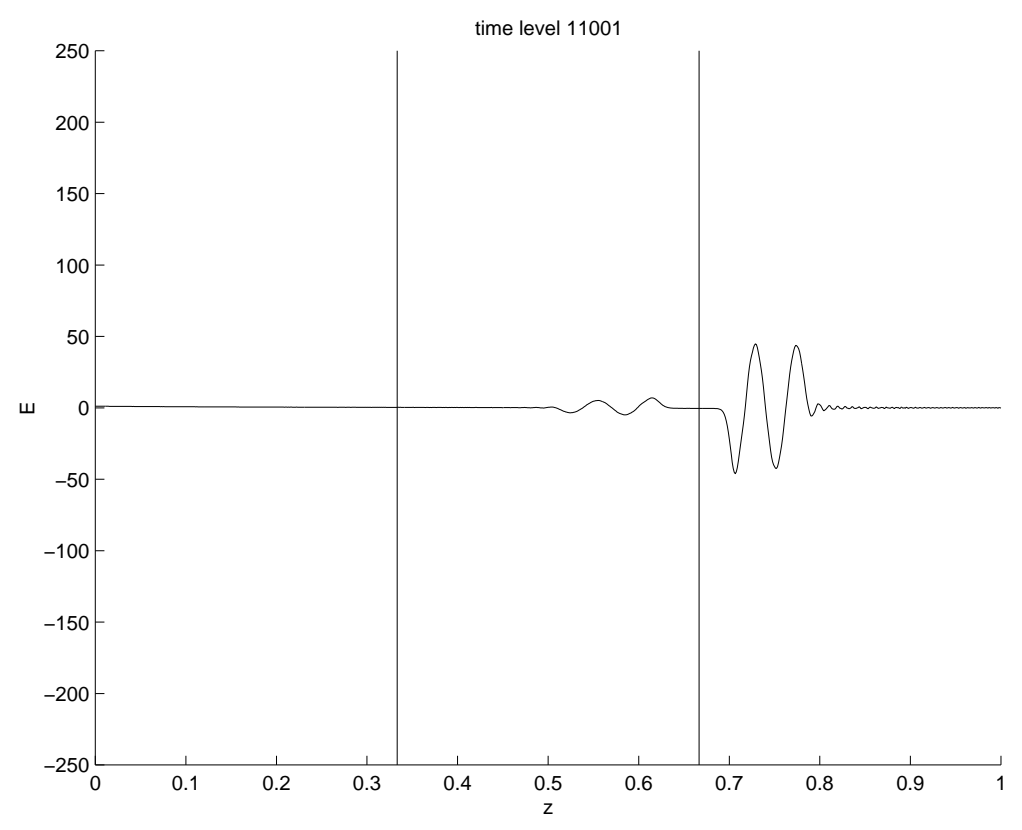

Figure 4: Example 1. The electronic wave in the part of fluid $\left[z_{2}, 1\right]$ where there is an acoustic wave. A reflected wave generated from the acoustic beam is seen in $\left[z_{1}, z_{2}\right]$.

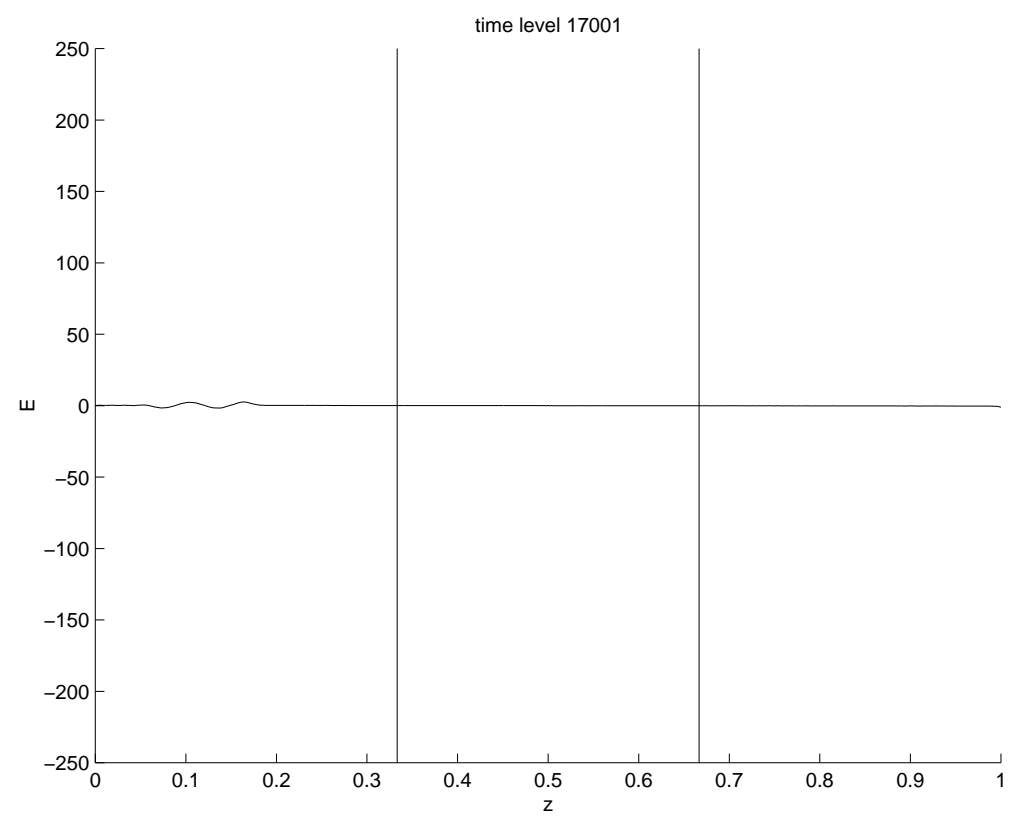

Figure 5: Example 1. The reflected wave from the acoustic beam approaches the left boundary. 


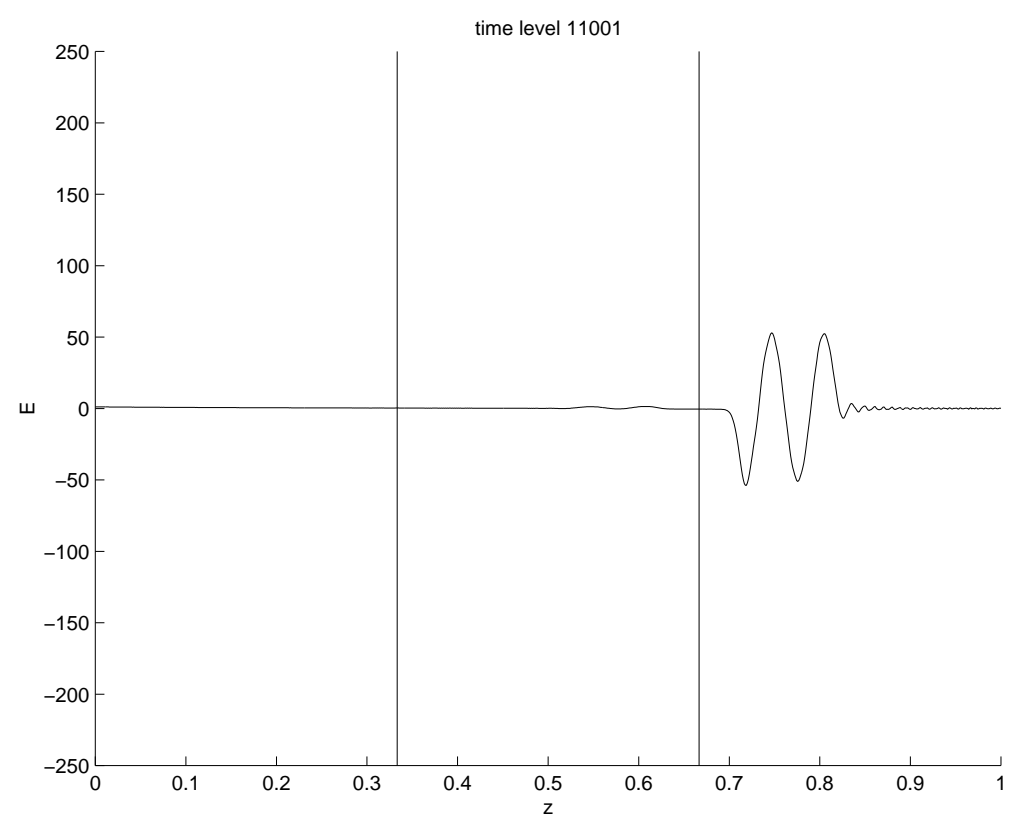

Figure 6: Example 2. The electronic wave in the part of the fluid where there is an acoustic wave. A rather weak reflected wave is generated from the acoustic beam at a lower frequency.

Example 3: A smaller relaxation time $\tau$ is used in this example, all other parameters are the same those in Example 1 (see Table 3). From Figure 7, 8, and 9 we can see that the transmitted electronic wave into the fluid is weak, with most of the electronic wave reflected from the interface between air and fluid.

\begin{tabular}{|c|c|c|c|}
\hline$t_{f}$ & $4.0 \times 10^{-10}$ & $\tau$ & $8.1 \times 10^{-12}$ \\
\hline$\epsilon_{\infty}$ & 5.5 & $\epsilon_{s}$ & 78.2 \\
\hline$\sigma$ & $1.0 \times 10^{-5}$ & $\kappa$ & 10.0 \\
\hline$\omega_{p}$ & $\pi \times 10^{7}$ & $\omega_{s}$ & $\pi \times 10^{10}$ \\
\hline$\Delta t$ & $t_{f} / 1600$ & $h$ & $1 / 900$ \\
\hline
\end{tabular}

Table 3: Parameters used for example 3. 


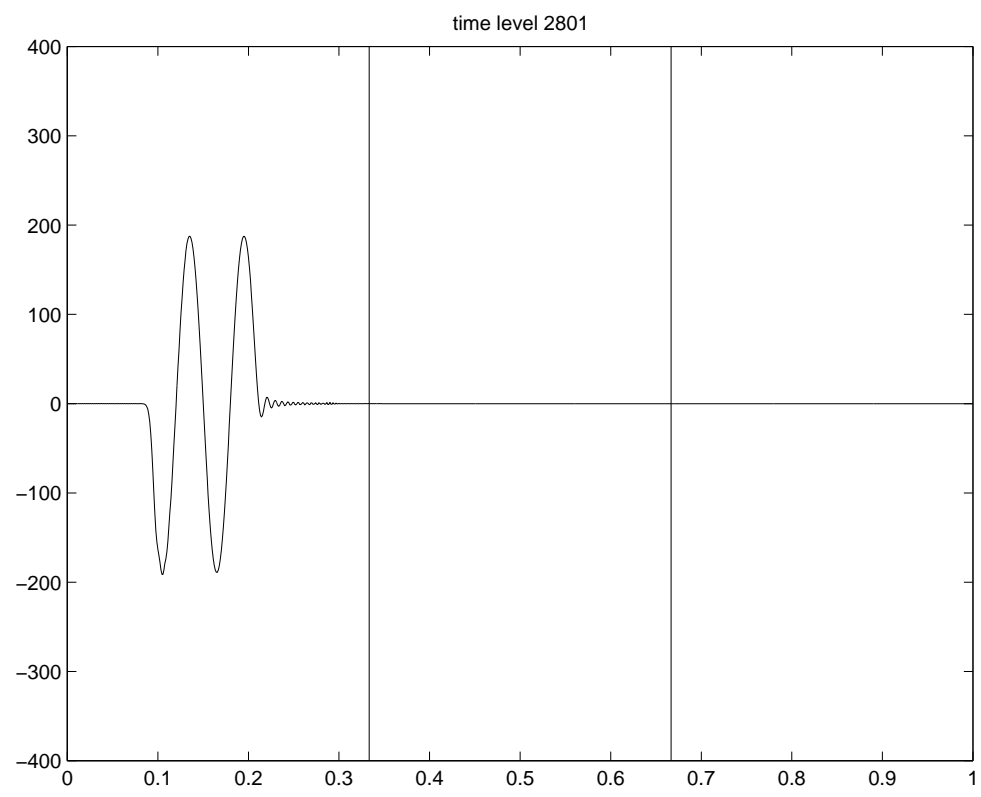

Figure 7: Example 3. The electronic wave before it reaches fluid.

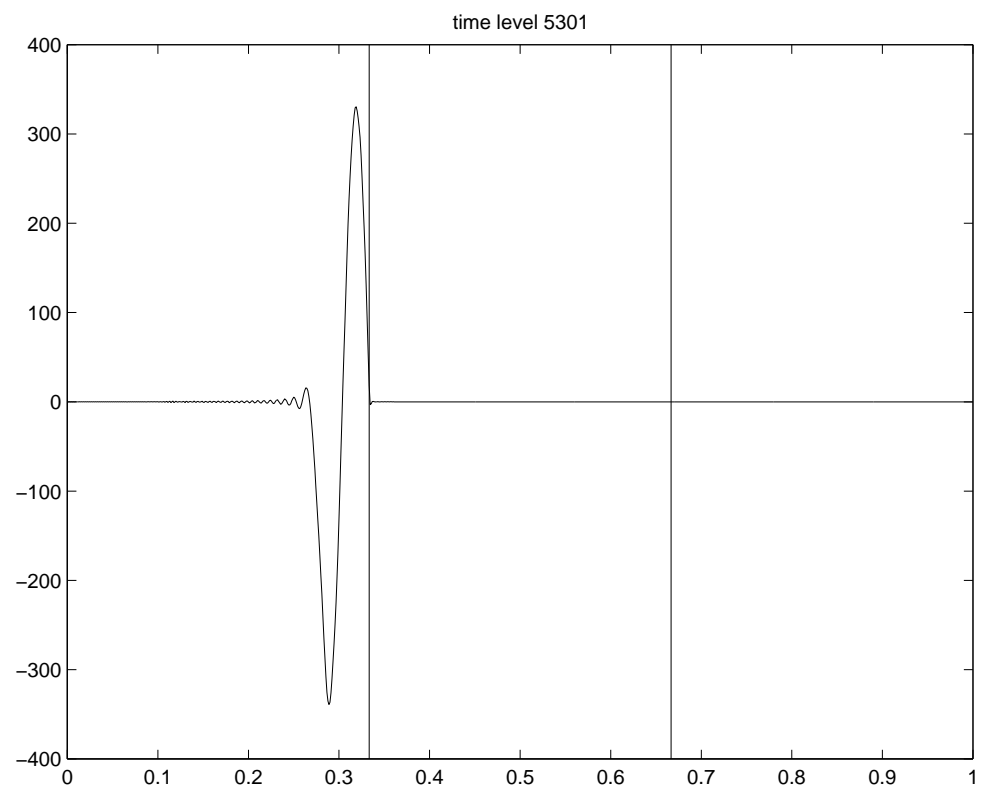

Figure 8: Example 3. Transmitted electronic wave into the fluid is weak. 


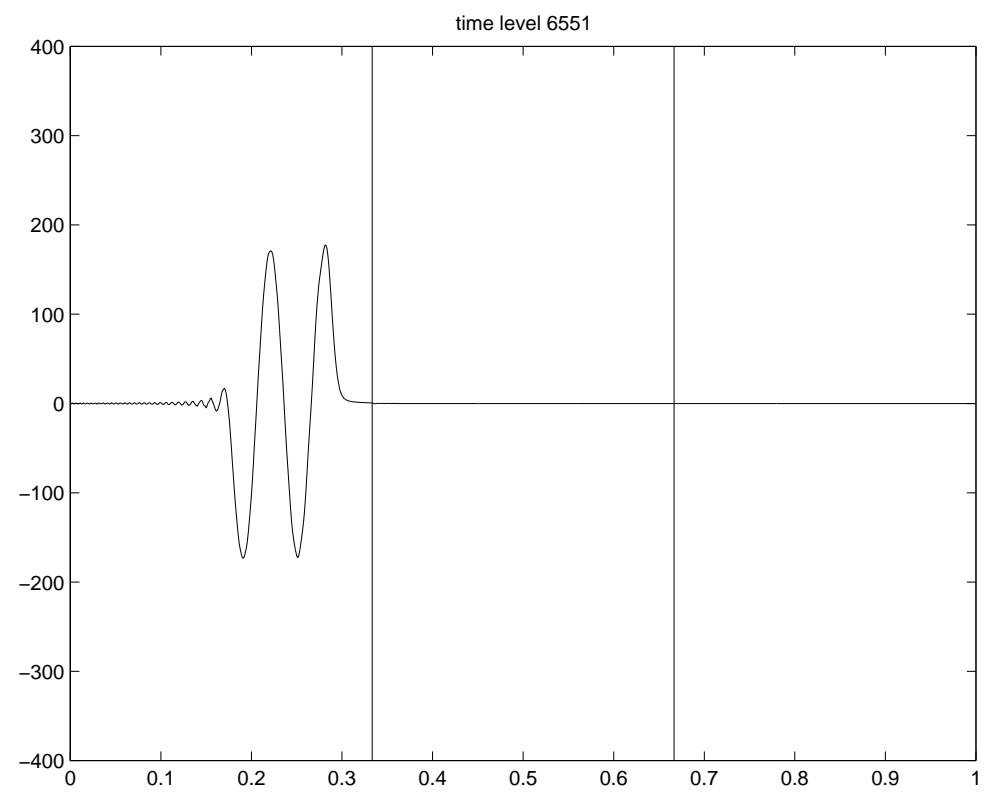

Figure 9: Example 3. Most of the wave is reflected back into the domain filled with air.

\section{Identification of material depth}

In this section, we consider the problem of using the signal $E(t, 0)$ collected at the left boundary $z=0$ to estimate the material depth. To be specific, we assume that all the physical parameters other than the depth of the material are given (these can be estimated using the first reflected waves from the interface at $z_{1}$, see $[2,3]$ ), and the interface of the material closer to the source (which is also at $z=0$ ) is fixed at $z=z_{1}$. As before, we denote the position of the interior interface between the fluid and acoustic domain by $z_{2}$. After an electromagnetic wave is sent to the material, reflected waves will be generated at the two interfaces of the material that will propagate back to the left boundary where the wave is generated. Intuitively, the difference between the times when the wave reflected from the first interface and that from the second interface reach the left boundary depends on the depth. Hence we shall attempt to estimate the depth of the material from this difference.

We first need a procedure to detect the time when a wave reflected from the interface reaches the left boundary. Figure 10 depicts a typical data function $E(t, 0)$. Note that the data function is essentially zero except in three subintervals. We first considered using the velocity $E_{t}(t, 0)$ to estimate $z_{2}$. We expect the function $E_{t}(t, 0)$ to become zero after the source has been turned off for a while. Then $E_{t}(t, 0)$ becomes nonzero when the wave reflected from the first interface arrives at the left boundary. This is followed by a period of time when $E_{t}(t, 0)$ becomes zero again. Afterwards, $E_{t}(t, 0)$ becomes nonzero due to the arrival of the wave reflected from the second interface. Figure 11 presents a typical plot of 
$\left|E_{t}(t, 0)\right|$. To aid in our estimation procedure, we define $T_{1}$ to be the first time when

$$
E_{t}\left(T_{1}, 0\right)>C_{1}, \quad T_{1}>C_{2},
$$

where both constants $C_{1}$ and $C_{2}$ can be determined from the measurement of $E(t, 0)$. The time $C_{2}$ should be larger than the time within which the source is generated. The constant $C_{1}$ should be chosen large enough to distinguish the change in data due to measurement error. Since $z_{1}$ is assumed to be fixed in the identification of depth, $T_{1}$ should be independent of $z_{2}$. We next let $T_{2}$ be the first time such that

$$
E_{t}\left(T_{2}, 0\right)>C_{3}, \quad T_{2}>T_{1}+C_{4} .
$$

The constant $C_{3}$ can be chosen in a way similar to that for $C_{1}$, which we can simply take $C_{3}=C_{1}$. The value of $C_{4}$ should be chosen so that the wave reflected from the first interface has passed the left boundary at the time $t=T_{2}+C_{4}$. Clearly $T_{2}$ depends on the position of the second interface, and we denote it by $T_{2}=T_{2}\left(z_{2}\right)$. Then we define a function of $z_{2}$ as the difference of these two characteristic times:

$$
L\left(z_{2}\right)=T_{2}\left(z_{2}\right)-T_{1},
$$

and our identification problem leads to looking for a value $z_{2}$ such that

$$
L\left(z_{2}\right)=L\left(z_{2}^{*}\right)
$$

where $z_{2}^{*}$ is the true position of the second interface of the material from which the data is collected. Note that $z_{2}^{*}$ is unknown, but $L\left(z_{2}^{*}\right)$ can be generated from the data.

To see the behavior of $L\left(z_{2}\right)$, we calculated its values for various $z_{2}$ in the neighborhood of $z_{2}^{*}$. A typical plot of $L\left(z_{2}\right)$, given in Figure 12, suggests that $L\left(z_{2}\right)$ acts almost like an affine function in the neighborhood of $z_{2}^{*}$. In this case, the secant method is a good candidate for computing $z_{2}$ from (4.21).

Example 4: We assume that the true position of the second interface is at $z_{2}^{*}=2 / 3$. The data $E(t, 0)$ was generated by the finite element scheme presented in Section 2 with $z_{2}=z_{2}^{*}$ and other parameters listed in Table 4 . The plot for the data of $E(t, 0)$ is in Figure 10, and the absolute value of its numerical derivative is plotted in Figure 11. From these plots, we decided to choose the constants $C_{1}, C_{2}, C_{3}, C_{4}$ as follows:

$$
C_{1}=C_{3}=4.5 \times 10^{10}, \quad C_{2}=2.0 \times 10^{-9}, \quad C_{4}=1.25 \times 10^{-9} .
$$

With these constants, we can find that

$$
L\left(z_{2}^{*}\right)=2.2165 \times 10^{-9} .
$$

Then we used the secant method to solve iteratively for $z_{2}$ in

$$
L\left(z_{2}\right)=2.2165 \times 10^{-9},
$$




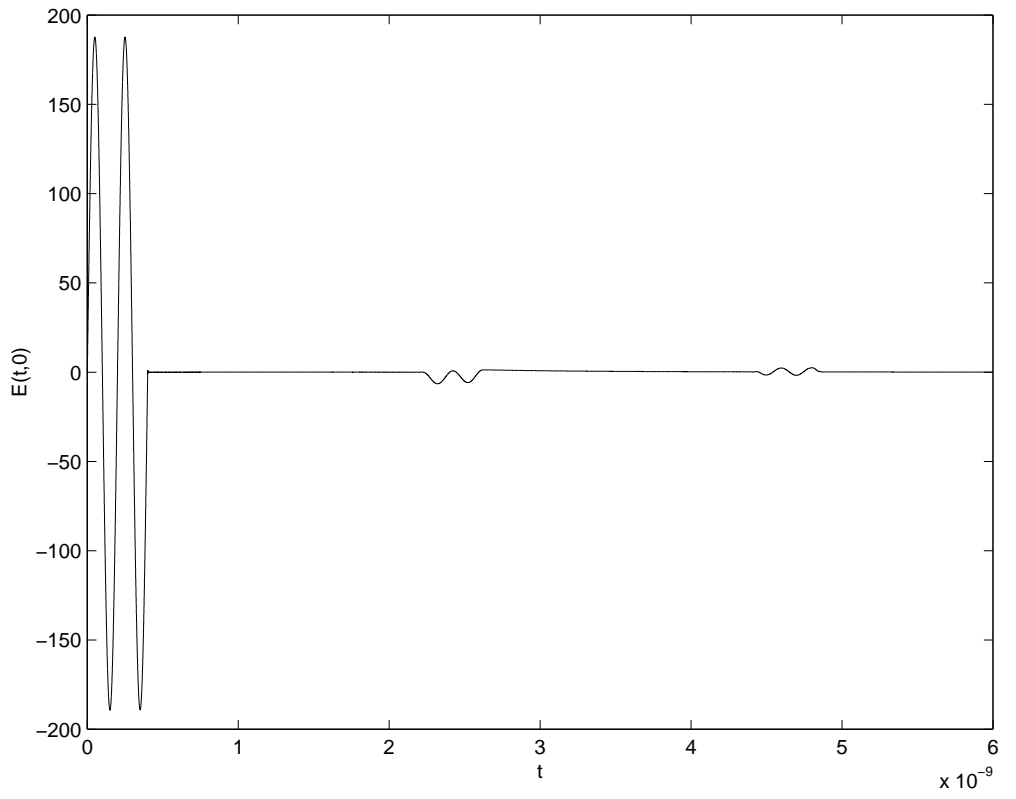

Figure 10: A typical data function.

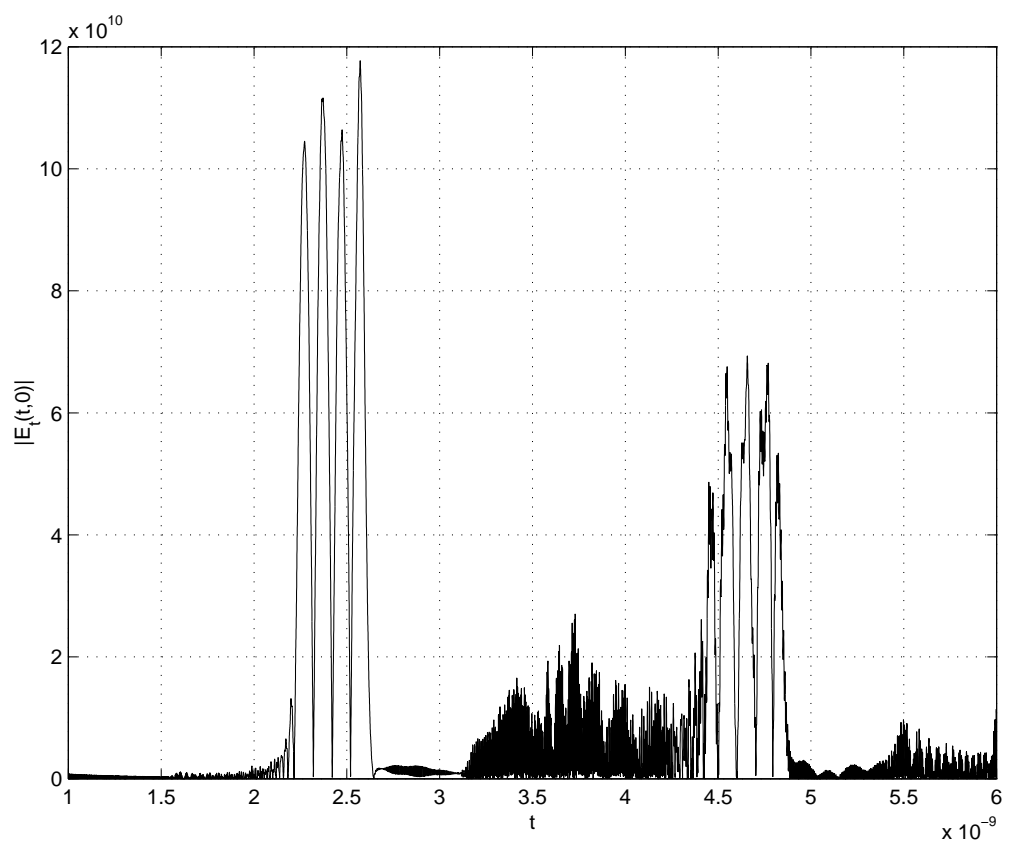

Figure 11: A typical plot of $\left|E_{t}(t, 0)\right|$. 


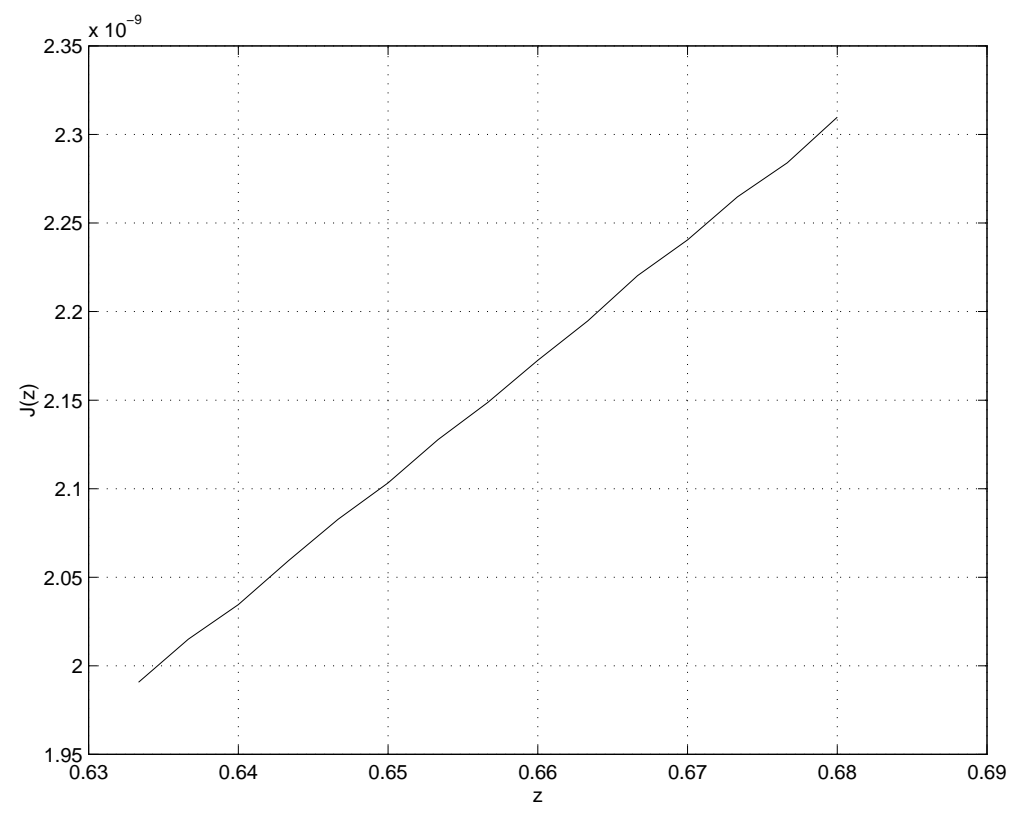

Figure 12: A typical plot of $L\left(z_{2}\right)$ in the neighborhood of $z_{2}^{*}$.

\begin{tabular}{|c|c|c|c|}
\hline$t_{f}$ & $4.0 \times 10^{-10}$ & $\tau$ & $3.162277660168379 \times 10^{-8}$ \\
\hline$\epsilon_{\infty}$ & 5.5 & $\epsilon_{s}$ & 78.2 \\
\hline$\sigma$ & $1.0 \times 10^{-5}$ & $\kappa$ & 10.0 \\
\hline$\omega_{p}$ & $\pi \times 10^{7}$ & $\omega_{s}$ & $\pi \times 10^{10}$ \\
\hline$\Delta t$ & $t_{f} / 1600$ & $h$ & $1 / 1800$ \\
\hline
\end{tabular}

Table 4: Parameters used for Example 4.

and obtained the following approximation to the exact position of the second interface:

$$
\hat{z}_{2}=0.66653017606683 \approx z_{2}^{*}=2 / 3 \text {. }
$$

We remark that in the computation of $\hat{z}_{2}$, we used $h=1 / 900$ and $\tau=t_{f} / 1600$ in the initial boundary value problem.

We observe that the above scheme appears to be a reasonable approach only if we can have a dependable measurement for the velocity $E_{t}(t, 0)$. This approach most likely will not work if numerical differentiation must be used to obtain an estimate of $E_{t}(t, 0)$ from a measurement of $E(t, 0)$ with noise. This is due to the well known catastrophic behavior encountered in using numerical differentiation on error-polluted data. Even a very small amount of error in the data for $E(t, 0)$ will make the estimate of $E_{t}(t, 0)$ generated by numerical differentiation meaningless. For example, if we pollute the data given in the previous example by a uniformly distributed random relative error with a magnitude only $5 \%$ of that of the data, then the 


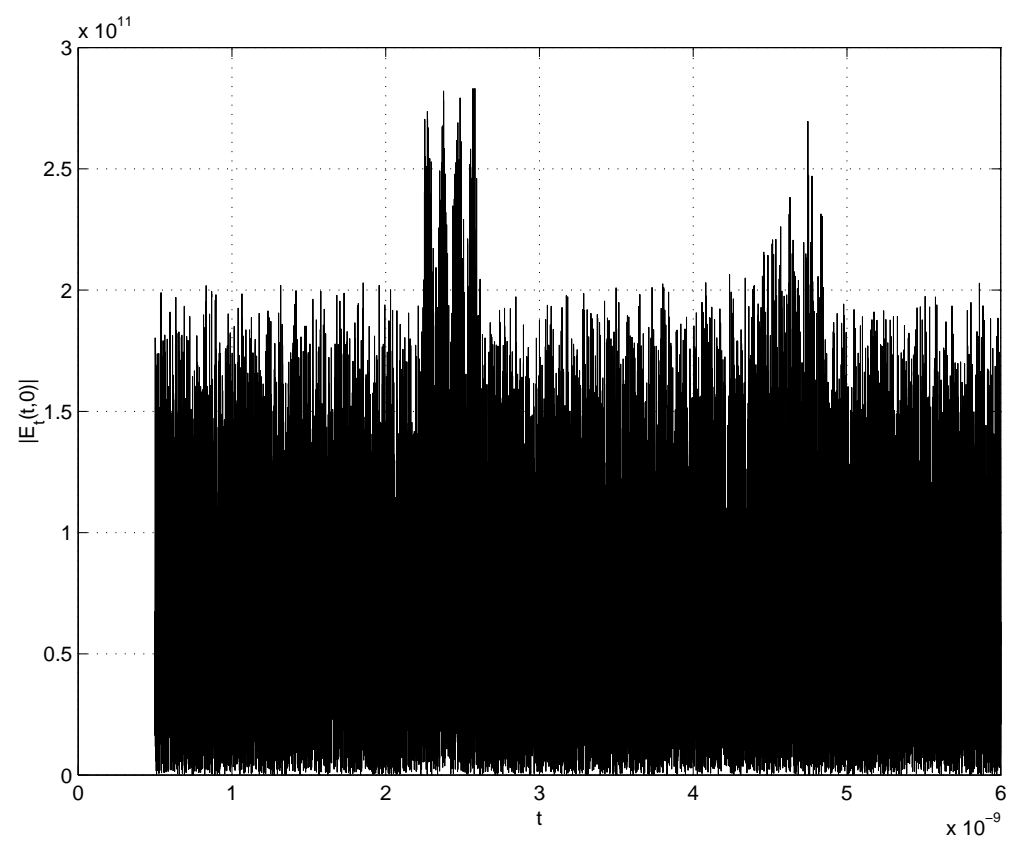

Figure 13: A typical plot of an estimated $\left|E_{t}(t, 0)\right|$ generated from numerical differentiation on a measurement of $E(t, 0)$ with a random error whose magnitude is only $5 \%$ of the true data.

$E_{t}(t, 0)$ plotted in Figure 13 generated by numerical differentiation does not give any useful information about the time lag between the two reflected waves.

On the other hand, the pulse signal $E(t, 0)$ itself appears to be rather robust with respect to the random noise from the point of view of indicating the time lag between the reflected waves from the two interfaces. Figure 14 is a plot of the absolute value of the data for $E(t, 0)$. Adding a uniformly distributed random error at the $5 \%$ level yields data plotted in Figure 15 from which we can still easily discern the times when the two reflected waves arrive at the left boundary. Hence we introduce another quantity to describe the time lag from a measurement of $E(t, 0)$ as follows.

We define $T_{1}$ to be the first time such that

$$
E\left(T_{1}, 0\right) \geq C_{1}, \quad T_{1}>C_{2},
$$

where both constants $C_{1}$ and $C_{2}$ can be determined from the measurement of $E(t, 0)$. The value of $C_{2}$ should be larger than the time during which the source is generated. The constant $C_{1}$ should be chosen sufficiently large so as to distinguish the change in data due to measurement error. The time $T_{1}$ should be independent of $z_{2}$ since $z_{1}$ is assumed to be fixed in the estimation of depth. We let $T_{2}$ be the first time such that

$$
E\left(T_{2}, 0\right) \geq C_{3}, \quad T_{2}>T_{1}+C_{4}
$$


The constant $C_{3}$ can be chosen in a way similar to that for $C_{1}$ (we can even simply let $C_{3}=C_{1}$ ). The constant $C_{4}$ should be chosen so that the wave reflected from the first interface has passed the left boundary at the time $t=T_{2}+C_{4}$. As expected $T_{2}$ depends on the position of the second interface, and we denote it by $T_{2}=T_{2}\left(z_{2}\right)$. Then we define a function of $z_{2}$ as the difference of these two characteristic times:

$$
L\left(z_{2}\right)=T_{2}\left(z_{2}\right)-T_{1}
$$

Obviously, the construction procedure for $L\left(z_{2}\right)$ is similar to the one associated with data $E_{t}(t, 0)$, but no differentiation is used. As before, we define the solution to the identification problem as a quantity $z_{2}$ that satisfies

$$
L\left(z_{2}\right)=L\left(z_{2}^{*}\right)
$$

Since this new time lag function of $z_{2}$ also appears to behave linearly in the neighborhood of the exact location of the second interface $z_{2}^{*}$ (see Figure 16), we again believe that the secant method is a good candidate for computing $z_{2}$ from (4.22).

Example 5: The function $L\left(z_{2}\right)$ just introduced is also rather insensitive to noise in the data. See Table 5 for its behavior with respect to the random error with a uniform distribution, and Table 6 for its behavior with respect to the random error with a normal distribution.

\begin{tabular}{|c|c|}
\hline Noise Level & $L\left(z_{2}^{*}\right)$ by datum with noise \\
\hline 0 & $2.223 \times 10^{-9}$ \\
\hline $1 \%$ & $2.22325 \times 10^{-9}$ \\
\hline $5 \%$ & $2.2225 \times 10^{-9}$ \\
\hline $10 \%$ & $2.2225 \times 10^{-9}$ \\
\hline $15 \%$ & $2.22275 \times 10^{-9}$ \\
\hline $20 \%$ & $2.22225 \times 10^{-9}$ \\
\hline
\end{tabular}

Table 5: Measure of sensitivity of $L\left(z_{2}\right)$ to the noise with a uniform distribution in the data.

Here we used the finite element solution to generate a data for $E(t, 0)$ with $z_{2}^{*}=2 / 3$ and other parameters listed in Table 4 . The plot for the data of $E(t, 0)$ is in Figure 10 , and the absolute value of the two reflected waves received at the left boundary is plotted in Figure 14. From these plots, we decide to choose the constants $C_{1}, C_{2}, C_{3}, C_{4}$ for the definition of $L\left(z_{2}\right)$ as follows:

$$
C_{1}=C_{3}=1.0, \quad C_{2}=2.0 \times 10^{-9}, \quad C_{4}=1.75^{-9} .
$$

We perturbed this data by random numbers with various noise level, and used the error polluted data to generate values of $L\left(z_{2}^{*}\right)$ in Table 5 and Table 6 . Note that $L\left(z_{2}^{*}\right)$ changes little even with the data at the $20 \%$ noise level. 


\begin{tabular}{|c|c|}
\hline Standard deviation & $L\left(z_{2}^{*}\right)$ by datum with noise \\
\hline 0 & $2.223 \times 10^{-9}$ \\
\hline 0.01 & $2.2235 \times 10^{-9}$ \\
\hline 0.05 & $2.222 \times 10^{-9}$ \\
\hline 0.1 & $2.2203 \times 10^{-9}$ \\
\hline 0.15 & $2.2258 \times 10^{-9}$ \\
\hline 0.2 & $2.219 \times 10^{-9}$ \\
\hline
\end{tabular}

Table 6: Measure of sensitivity of $L\left(z_{2}\right)$ to the noise with a normal distribution in the data.

Example 6: The estimation procedure for the depth $z_{2}^{*}$ based on this new time lag function works well with data polluted with random errors at various levels as seen Table 7. Even the data with $10 \%$ noise yields a good approximation to the location of the second interface. Note that in the computation of $\hat{z}_{2}$, we used the physical parameters listed in Table 4 , but we used $h=1 / 900$ and $\Delta t=t_{f} / 1600$ to solve the initial boundary value problem, and the exact location of the second interface is $z_{2}^{*}=2 / 3$.

\begin{tabular}{|c|c|}
\hline Noise Level & $\hat{z}_{2}$ by data with noise \\
\hline 0 & 0.66632745773773 \\
\hline $1 \%$ & 0.66632745773773 \\
\hline $5 \%$ & 0.66640117743327 \\
\hline $10 \%$ & 0.66629059787037 \\
\hline
\end{tabular}

Table 7: The estimation procedure is rather robust with respect to the random noise in the data. Uniformly distributed random errors were used in these computations. 


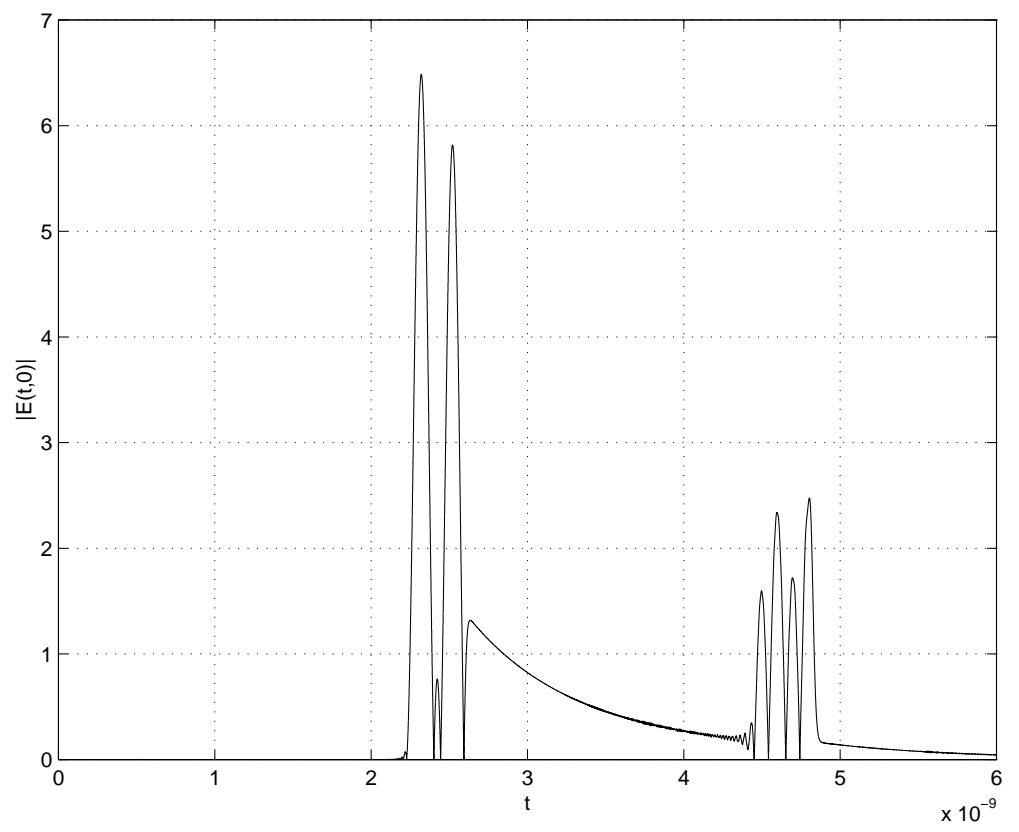

Figure 14: A plot of $E(t, 0)$, uncorrupted

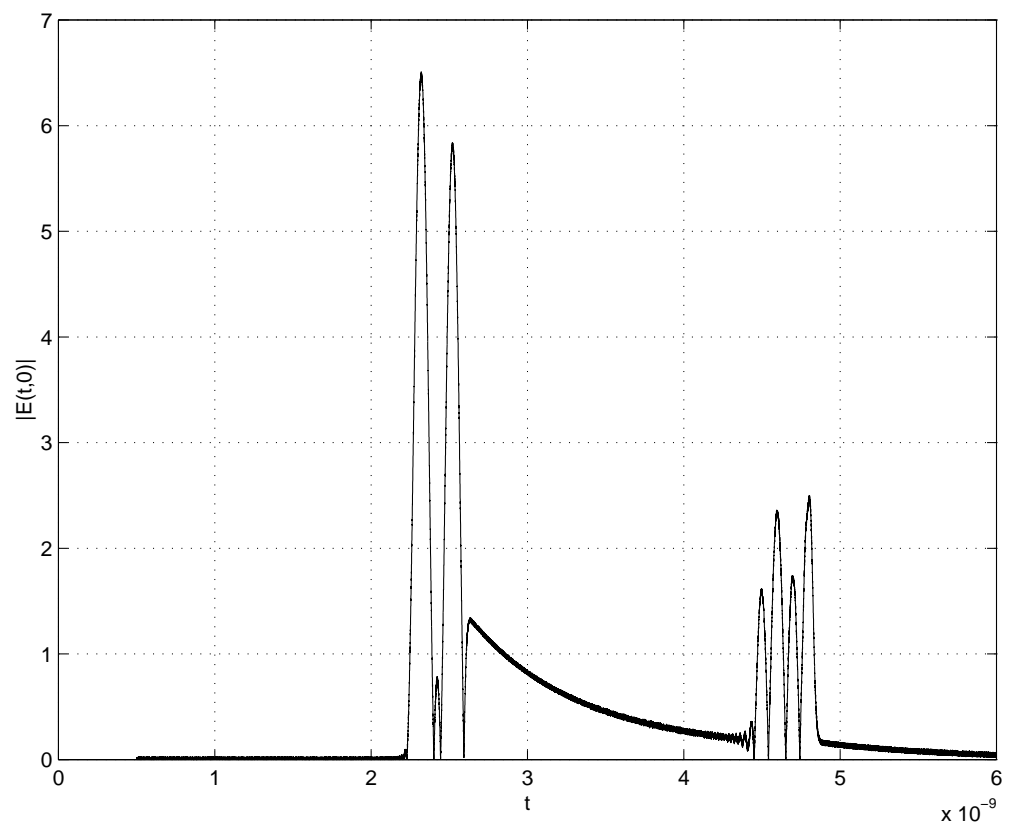

Figure 15: A plot of $E(t, 0)$, corrupted with $5 \%$ random relative error. 


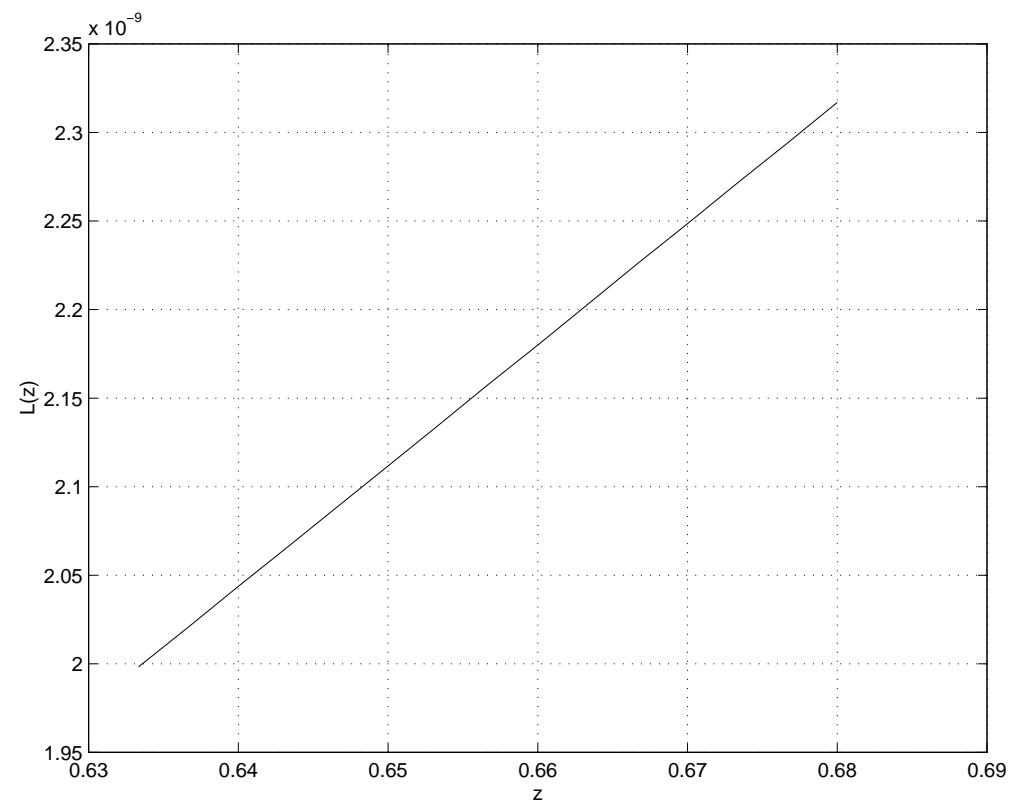

Figure 16: A typical plot of $L\left(z_{2}\right)$ in the neighborhood of $z_{2}^{*}=2 / 3$.

\section{Concluding remarks}

In the preliminary investigations reported on in this note, we have demonstrated the potential to employ internal acoustic fields as reflectors for electromagnetic probes in the interrogation of dielectric media. The associated inverse problems are based on time domain formulation of the acoustooptic signals in the media. Encouraged by these early findings, our efforts are continuing with more involved electric polarization models as well as with models of the acoustooptic interaction that are closer to physical reality.

\section{Acknowledgment}

The authors are most grateful to Dr. Richard Albanese for his continued encouragement in the direction of the research reported here. We are equally indebted to him for specific technical discussions throughout the course of our efforts. We are also pleased to acknowledge helpful discussions with Michael Buksas. In particular, we benefited immensely in comparing results from the solution methods reported here with those based on his time domain formulations reported on in $[2,3]$.

\section{References}

[1] R.A. Albanese, R.L. Medina, and J.W. Penn. Mathematics, medicine and microwaves. 
Inverse Problems, $\underline{10}$ (1994), 995-1007.

[2] H.T. Banks and M.W. Buksas. Electromagnetic interrogation of dielectric materials. Technical report, Center for Research in Scientific Computation, North Carolina State University, to appear.

[3] H.T. Banks, M.W. Buksas, and Y.Wang. A time domain formulation for identification in electromagnetic dispersion. Technical Report CRSC-TR96-30, NCSU, Journal of Math. Systems, Estimation and Control, $\underline{8}(1998), 257-260$.

[4] H.T. Banks and T. Lin. Models of acousto-optic interactions in dielectric media. Technical report, Center for Research in Scientific Computation, North Carolina State University, to appear.

[5] L. Brillouin. Diffusion de la lumiére et des rayons x par un corps transparent homogéne influence de l'agitations thermique. Annales de Physique (French), 17(1922), 88-122.

[6] V.V. Daniel. Dielectric Relaxation. Academic Press, New York, 1967.

[7] P.K. Das and C.M. DeCusatis. Acoustio-Optic Signal Processing: Fundamentals 68 Applications. Artech House, Inc., Boston, 1991.

[8] A. Korpel. Acousto-optics. Optical Engineering, Vol. 57. Marcel Dekker, Inc., New York, second edition, 1997.

[9] P.P. Morse and K.U. Ingard. Theoretical Acoustics. International Series in Pure and Applied Physics. McGraw-Hill Book Company, New York, 1968.

[10] J. Xu and R. Stroud. Acousto-Optic Devices. John Wiley \& Sons, Inc., New York, 1992. 